Cahiers d'études italiennes

Intuizione e forma. André Jolles: vita, opere, posterità

\title{
Le Fiabe teatrali di Carlo Gozzi nell'interpretazione di Jolles
}

Les Fiabe teatrali de Carlo Gozzi dans l'interprétation de Jolles

Carlo Gozzi's Tales for the Theatre in the Interpretation of Jolles

\section{Andrea Penso}

\section{OpenEdition}

\section{Journals}

Edizione digitale

URL: http://journals.openedition.org/cei/3203

DOI: $10.4000 /$ cei.3203

ISSN: 2260-779X

\section{Editore}

UGA Éditions/Université Grenoble Alpes

Edizione cartacea

Data di pubblicazione: 30 décembre 2016

Paginazione: 137-147

ISBN: 978-2-84310-337-7

ISSN: 1770-9571

\section{Notizia bibliografica digitale}

Andrea Penso, «Le Fiabe teatrali di Carlo Gozzi nell'interpretazione di Jolles», Cahiers d'études italiennes

[Online], 23 | 2016, online dal 23 janvier 2017, consultato il 26 mars 2021. URL: http:// journals.openedition.org/cei/3203 ; DOl: https://doi.org/10.4000/cei.3203 


\title{
LE FIABE TEATRALI DI CARLO GOZZI NELL'INTERPRETAZIONE DI JOLLES
}

\author{
Andrea Penso \\ Università di Padova
}

Nel suo saggio su La fiaba nella letteratura occidentale moderna (1922I923), André Jolles si sofferma ampiamente sulle Fiabe teatrali di Carlo Gozzi. I lavori di Gozzi erano già molto noti in area germanica a partire dagli anni '8o del Settecento. Ma fu soprattutto grazie all'opera dei fratelli Schlegel, di Hitzig e di Hoffmann che la fortuna del commediografo veneziano nell'Europa settentrionale crebbe sensibilmente. Friedrich Schlegel era un grande ammiratore del teatro gozziano, del quale aveva rilevato tutta l'originalità. Riteneva infatti che la commedia con maschere rimessa in auge dal Veneziano fosse l'unico genere apprezzabile dalle persone che cercavano la novità a teatro, e per le stesse ragioni rimpiangeva l'abbandono da parte del teatro italiano della nuova via inaugurata da Gozzi nelle Fiabe teatrali con la compresenza di due generi popolari: da una parte la fiaba, connotata da una intrinseca poeticità, dall'altra la Commedia dell'Arte, le cui maschere incarnavano l'ironia che mette in ridicolo la parte poetica. Le Fiabe teatrali erano per Schlegel emblemi di un uso estroso e spregiudicato delle modalità fiabesche ed erano state considerate ingiustamente alla stregua di produzioni stravaganti, e non invece come squisiti prodotti di quella buffoneria forse un po' malinconica così cara al Romanticismo tedesco. L'interesse di Schlegel per le «fole sceniche» di Gozzi era infatti suscitato proprio dalla loro mescolanza di serio e faceto, cui corrispondeva da un lato un accostamento di piani e registri linguistici con diversa funzione comunicativa, dall'altro la compresenza di stili e di generi differenti.

Non per nulla, il tipo di ironia adoperato da Gozzi avrà una parte cospicua nel processo di «romantizzazione» della letteratura, e le Fiabe teatrali saranno un indiscusso modello di un nuovo modo di intendere questo tipo di produzione letteraria, ben presto esteso all'area semantica 
e simbolica dell'Arabeske e del Capriccio. Anzi, tenendo conto del fatto che il termine Arabeske assume, nel linguaggio schlegeliano (soprattutto nel Brief über den Roman e nella Rede über die Mythologie) e quindi della maggior parte dei romantici tedeschi, la connotazione preziosa e lieve dell'ornamento, del ghirigoro orientaleggiante, ma anche del sogno, della fantasia come atto liberatorio e gratuito, si comprende anche quanto il gusto per l'Arabeske e il Capriccio abbia a che fare con il diffondersi di una "Gozzische Manier» ${ }^{\mathrm{e}}$ e con il nascere di una letteratura fantastica, che avrà nella Prinzessin Brambilla (I82I) di Hoffmann uno dei modelli più fortunati e convincenti ${ }^{2}$. Nel dialogo Der Dichter und der Komponist, scritto ancora da Hoffmann nel I8I3, il personaggio del compositore Ludwig, parlando al poeta Ferdinand del meraviglioso nel teatro, cita la fiaba Il corvo di Gozzi come modello di un'azione drammatica da cui si potrebbe trarre un buon libretto d'opera romantica. L'impressione di Hoffmann dovette certo essere condivisa, se è vero che il teatro fiabesco di Gozzi divenne, dal Romanticismo all'epoca moderna, un ricco serbatoio di soggetti d'opera.

Studioso dei romantici e di Schlegel, Jolles non dovette essere sordo all'eco della fama e della popolarità di Gozzi, e tuttavia, nonostante questi autorevoli precedenti storico-critici, la presenza del commediografo veneziano nella ricognizione compiuta sulla «forma» della fiaba a partire dai nomi, certo meno sorprendenti, di Basile, La Fontaine, Perrault o delle Mille e una notte, appare tutt'altro che scontata, in ragione della natura fondamentalmente diversa delle Fiabe di Gozzi. Jolles stesso si premura infatti di avvertire il lettore che l'inserimento dell'elemento teatrale nel proprio scritto sulla fiaba costituisce solo in apparenza una deviazione dal canone di quella specifica «forma». Le prime battute del paragrafo dedicato a Gozzi ammettono la piena ascrivibilità delle rappresentazioni teatrali al genere della fiaba:

Il fatto che accanto a parvenze indipendenti e compiute vi siano altri aspetti letterari collegati alla fiaba, va da sé: frammenti di fiabe, motivi fiabeschi, umori fiabeschi si possono collezionare in grande quantità in epoche diverse o in differenti correnti letterarie. Così il teatro — soprattutto la commedia — appare spesso denso di tratti

I. R. S. Borello, L'amore delle tre meralance e i suoi travestimenti teatrali: da Gozzi a Sanguineti, in La letteratura degli italiani 4. I letterati e la scena, Atti del XVI Congresso nazionale Adi (Sassari-Alghero, I9-22 settembre 20I2), Roma, Adi editore, 20I4, p. 2.

2. È proprio questo romanzo-fiaba (o fiaba romanzata) a veicolare l'opera del Veneziano nei circuiti della grande letteratura europea. Nella Principessa Brambilla ritroviamo, infatti, alcuni degli aspetti più creativi e accattivanti delle fole gozziane, soprattutto per quanto riguarda il culto del fantastico e del capriccio, da intendere nell'accezione più bizzarra che il termine ha progressivamente assunto in letteratura, musica e pittura. L’opera cela, inoltre, l'aspirazione al recupero della Commedia dell'Arte, che viene riproposta a emblema della più totale e incondizionata libertà dell'artista. 
desunti dalla fiaba o a essa riconducibili. Si è ipotizzata più volte una relazione fra il teatro e la fiaba o tra la fiaba e il mimo, e all'occasione vi ritorneremo. Basti per ora l'esempio di Shakespeare: i suoi testi e quelli dei suoi contemporanei pullulano di tali motivi ${ }^{3}$.

Laccenno al teatro elisabettiano offre a Jolles il destro per sottolinearne il profondo legame con la novella. È dunque evidente come, nel tentativo di risolvere il problema dell'origine della fiaba, Jolles si orienti subito verso la letteratura. Il critico non nega, certamente, l'importanza fondamentale dell'oralità per la trasmissione delle fiabe, ma la relativizza. Siamo davvero sicuri, si chiede Jolles, che questi racconti fossero solamente ascoltati e non letti? Lo studioso olandese suppone dunque un accorciamento delle distanze tra la «letteratura dei libri» e la «tradizione popolare»:

Siamo inclini a considerare tutto ciò che troviamo presso il "popolo» come molto antico, primitivo o addirittura originario. E tuttavia viviamo in una civiltà che dal punto di vista storico e sociale si compone di livelli diversi, dove ciò che appartiene a uno strato si comunica poi agli altri secondo un rapporto di reciprocità. A guardar bene, si notano tra quelle che sono definite "canzoni popolari» opere di grandi compositori, e d'altro canto i compositori si fondano sulle cosiddette melodie popolari [...]. Non potrebbe avvenire lo stesso in rapporto a quello che noi chiamiamo da un lato la letteratura dei libri e dall'altro la tradizione popolare? ${ }^{4}$

Nell'ambito di una ricognizione di genere, tra fiaba, novella e teatro, la letteratura offre più stabili punti d'appoggio (essendo in sé compiuta anche se estremamente variegata) rispetto alla tradizione orale, la quale deve in ogni caso servirsi del filtro della scrittura, risultando a volte poco omogenea e scarsamente decifrabile a causa della complessa molteplicità delle sue implicazioni. Il genere letterario della novella chiamato in causa da Jolles intratteneva rapporti molto stretti con il teatro dell'epoca elisabettiana, così stretti che il tentativo di circoscrivere i confini dell'elemento prettamente drammatico rischierebbe secondo il critico di portare a una mancata comprensione di quelle opere teatrali dal loro interno. Jolles giunge anzi ad affermare che molti testi teatrali elisabettiani potrebbero essere definiti attraverso la nozione di «novella visibile»s. Nella prima parte del saggio, durante la ricognizione volta a comprendere da dove provengono le fiabe più note, proprio la novella era stata indicata come il principale veicolo letterario, svincolato dall'aleatorietà della tradizione orale, di

3. A. Jolles, I travestimenti della letteratura. Saggi critici e teorici (I897-1932), S. Contarini (a cura di), Milano, Bruno Mondadori, 2003, p. I85.

4. Ivi, p. II7.

5. Per il concetto di "visibilità» si veda il saggio di Jolles sopra La visione d'amore nella Vita nuova, in Ivi, p. 23 . 
elementi fiabeschi. Elementi che, anche se certamente eccezionali rispetto all'intrinseco realismo caratteristico della novella, furono di importanza fondamentale nella divulgazione di un gusto diverso, in qualche modo «nuovo» ${ }^{6}$.

A questo punto, è opportuno riflettere brevemente sulle relazioni che intercorrono tra i tre generi chiamati in causa sin qui. Se la novella diviene a un certo punto veicolo della fiaba, ed è legata a doppio filo al teatro, è chiaro come proprio il teatro amplifichi quegli elementi fiabeschi che nella novella avevano trovato diritto di cittadinanza. Secondo Jolles, anzi, è proprio il teatro il principale sostrato su cui attecchiscono i semi della fiaba: la commedia, in particolare, poteva per certi versi rinunciare all'esigenza di realismo cui la novella era stata piegata dalla tradizione toscana, che sulle scene non appariva più come un parametro vincolante, rendendo la suggestione dell'elemento fiabesco più viva e tangibile. Pur non potendo accertare nessun caso di commedie con discendenza diretta da fiabe, Jolles individua nel rapporto che intercorre tra il Winter's Tale di Shakespeare e il Pandosto di Greene, che di quello costituisce la fonte, un esempio della tendenza, a quell'altezza solamente in nuce, dell'opera teatrale a svuotare progressivamente il modello di partenza delle proprie istanze realistiche per accentuare invece l'elemento esotico e fiabesco.

Se il teatro di epoca elisabettiana aveva fatto intravvedere le potenzialità espressive della fiaba nei suoi contatti con i generi teatrali, è però solamente a partire dal Settecento che le cose mutano in maniera più decisa e iniziano a farsi sistema. Jolles individua almeno due fattori decisivi nell'evoluzione di questo processo. Il primo, che interessa relativamente il nostro discorso e a cui faremo cenno in maniera solamente cursoria, riguarda l'ingresso dell'Oriente negli scritti teatrali occidentali, e segnatamente francesi. La fiaba orientale compare a Parigi agli inizi del Settecento, inserendosi nella diatriba tra il teatro alto della Comédie française e quello di piazza del théâtre de la foire, sviluppatosi durante fiere ed eventi cittadini a margine del teatro ufficiale stabile, soprattutto a partire dal riversarsi nelle strade degli attori e del pubblico della Comédie italienne, chiusa per ordini superiori ${ }^{7}$. All'interno di questo teatro da strada, rudimentale

6. Il pioniere di questo nuovo corso viene individuato da Jolles in Giovan Francesco Straparola, autore della raccolta Le piacevoli notti e attivo a Venezia a partire dal 5530 . Alcuni dei suoi racconti non danno più, secondo Jolles, la sensazione di essere accaduti realmente, a causa della presenza dell'elemento soprannaturale come catalizzatore delle storie; d'altra parte, era stato l'influsso della tradizione toscana a imporre l'esigenza del realismo alla novella.

7. La rivalità tra la Comédie française e la Comédie italienne diede vita a una delle più importanti dispute nella drammaturgia del XVIII secolo. Il conflitto tra le due grandi istituzioni teatrali ha le sue radici nel sistema di privilegi di cui godeva la prima, e si aggravò con la francesizzazione del repertorio degli italiani, a partire 
ma estremamente ingegnoso, con influenze di Molière e della Commedia dell'Arte italiana, trovò una nicchia proprio la fiaba orientale, che ben si prestava a essere messa in scena in quei contesti più scanzonati, e che molta presa poteva avere sul gusto popolare, grazie anche al diffondersi del mimo come mezzo espressivo e comunicativo ${ }^{8}$. Si pensi per esempio alle oltre venti commedie che Alain-René Lesage compose tra il I7I3 e il I735, nelle quali il protagonista è un Arlecchino che, smessi i consueti panni della Commedia dell'Arte e abbandonati gli usuali imbrogli, viene collocato nei contesti più esotici: lo troviamo come re di Serendippo (Arlequin, roi de Serendib), come imperatore della Cina (Arlequin invisible chez le roi de la Chine), come gran visir e anche sultano (Arlequin grand vizir, Arlequin sultane favorite), fino addirittura ad assumere le connotazioni di un moderno Maometto (Arlequin Mahomet). In questo evidente influsso della tradizione orientale, Jolles scorge, se non una novità d'ingegno, almeno un impulso decisivo alla divulgazione degli elementi fiabeschi in ambito teatrale: il critico rileva infatti come l'influsso orientale sia penetrato in profondità nella cultura dell'epoca, sia nella letteratura alta che in quella bassa, costituendo la base anche del vaudeville e dell'opéra comique, che a loro volta parteciperanno alla diffusione di elementi fiabeschi.

Il secondo dei due poli cui si accennava sopra va invece individuato nella Venezia della seconda metà del Settecento. Jolles la descrive come una città «simile a un fiore appassito, a una danza di spettri», che "forse però cela al suo interno un cuore fervido e vitale, indizio di qualcosa di nuovo» ${ }^{9}$. Ci stiamo dunque avvicinando all'oggetto principale della nostra indagine, vale a dire Carlo Gozzi, che di quella Venezia, e del dibattito culturale che la animava, soprattutto in riferimento alla querelle sulla natura del buon teatro, fu indiscusso protagonista. Sostenitore della Commedia dell'Arte nel momento in cui le maschere tradizionali e il gioco a esse collegato erano allo stremo, Gozzi fu un risoluto conservatore dal punto di vista dell'ideologia letteraria. Jolles individua chiaramente i capisaldi del suo pensiero: fiero oppositore dell'Illuminismo e dell'Alessandrinismo francese importato da Martelli, Gozzi si collocava all'estremo opposto tanto rispetto al sentimentalismo di Pietro Chiari che al realismo di

dal I7I8. Tale rivalità si declinò secondo varie sfumature, sulla base di una agguerrita concorrenza, e si rivelò soprattutto sul piano istituzionale. In seguito, anche se la Comédie française rivendicava per sé la tradizione, il théâtre de la foire riuscì a mantenere un suo pubblico e a inaugurare una propria tradizione comica.

8. Jolles ritiene che le origini della fortuna popolare del mimo siano da ricercare nelle rigide ordinanze emanate contro la pratica del teatro di strada, per sfuggire alle quali poeti e frequentatori di fiere si ingegnarono facendo recitare ruoli diversi a un solo personaggio, o chiedendo al pubblico di accompagnare cantando i testi mostrati su un grande foglio bianco, mentre gli attori veri e propri mimavano i gesti.

9. A. Jolles, I travestimenti della letteratura, cit., p. I87. 
Goldoni, difendendo nella struttura della commedia teatrale l'importanza delle maschere, che rischiavano di venire cancellate dall'irruzione del quotidiano sulle scene. Com'è noto, la storia darà poi ragione alla linea di Carlo Goldoni, decretando il successo della sua riforma teatrale e delle novità da lui apportate, ma ciò non impedisce a Jolles di interrogarsi sulla bontà delle intuizioni di Gozzi, che pure risulteranno alla lunga sconfitte rispetto alle innovazioni goldoniane. La scelta conservatrice di Gozzi, si chiede infatti Jolles, era solamente il sogno di un intellettuale passatista, non più profeta in patria, oppure celava "qualcosa di nuovo, nella ricerca di un nucleo vitale e sempre presente all'interno delle forme antiche, che può essere temporaneamente dimenticato ma che non muore?»" ${ }^{10}$

Jolles pone la domanda ma sospende il giudizio, lasciandola implicitamente senza risposta. A essere degna di nota tuttavia è la precisazione posta dal critico olandese a mo' di corollario sulla questione, che viene in questo modo orientata verso un preciso orizzonte teorico. A conti fatti, Jolles sostiene che quella operata da Carlo Gozzi non fu altro che una sintesi tra il vecchio (si pensi alla difesa dei testi «fuori moda» composti da Giovanni Antonio Sacchi, che trasferì sul piano pratico la polemica teorica condotta contro la linea Goldoni-Chiari) e una sorta di ripresa personale e soggettiva di quella vecchia visione, che risultò in ultima analisi qualche cosa di nuovo grazie all'utilizzo e all'introduzione di materiali fantastici che decise di chiamare «fiabe». Grazie alla proposta di queste nuove forme, Gozzi poté prendere temporaneamente il sopravvento sui suoi avversari letterari, e le ragioni di questo successo, ancorché momentaneo, vanno ricercate a giudizio di Jolles nello stretto legame di parentela che è possibile intravvedere tra la Commedia dell'Arte e la fiaba. Come abbiamo visto, pur cursoriamente Jolles individuava proprio nel Settecento di Gozzi un momento di svolta per la fiaba, che cominciava ad assumere un ruolo di primo piano anche nella commistione con altre forme letterarie. In seno alla Commedia dell'Arte la fiaba poteva trovare la sua collocazione più felice: secondo Jolles il binomio poteva funzionare molto bene perché, come la commedia spoglia i personaggi delle loro peculiarità individuali e li riduce a tipi fissi, riconoscibili solo attraverso abiti e maschere, allo stesso modo la seconda riconduce fatti e accadimenti a motivi chiaramente riconoscibili. Ovviamente non è dato sapere con quanta cognizione di causa Gozzi avesse operato una sintesi di materiali così diversi eppure così affini, e lo stesso Jolles ne è consapevole, anche se osserva che, nell'introduzione all'edizione 
delle Fiabe teatrali del I772, Gozzi mostra di avere bene compreso il potere espressivo e comunicativo di materiali ritenuti spesso infantili e irreali, quando afferma che «un argomento inetto, falso e puerile, trattato con arte, apparecchio ed eleganza» può «impegnar gli animi, ridurli ad ascoltare con serietà e commuoverli fino al grado delle lacrime» ". Basterebbero queste osservazioni a far comprendere le ragioni per cui, a parere di Gozzi (e, di riflesso, di Jolles, che le ripropone a sostegno delle proprie argomentazioni), la fiaba poteva avere pieno diritto di cittadinanza nel genere teatrale: essa, come avviene appunto nel teatro, aveva tutte le carte in regola per movere, docere e ovviamente delectare, e poteva offrire in quella particolare congiuntura storico-culturale alcuni elementi di novità ideali per rinvigorire il genere oramai languente della Commedia dell'Arte. È questo il primo punto della sintesi teorica di Jolles riguardo alla mediazione tra fiaba e teatro nel Settecento. Lo studio condotto sulle dieci Fiabe, su cui tra poco ci soffermeremo, si traduce nel tentativo più ampio da parte di Jolles di armonizzare sul piano teorico le spinte centrifughe degli esotismi delle fiabe soprattutto orientali, sempre più popolari e accolti dunque anche da Gozzi, e la volontà di rinvigorire il teatro partendo da una riaffermazione della Commedia dell'Arte e di una posizione anti-illuministica, arricchite dall'impulso proveniente dalle fiabe stesse.

La seconda parte del saggio jollesiano su Gozzi consiste in una analisi delle Fiabe teatrali, volta in prima battuta all'individuazione di talune fonti sottese alle commedie dell'autore veneziano, e in secondo luogo alla determinazione della chiave di volta che consenta di spiegare la dialettica sussistente tra fiaba e teatro. Ciò che interessa Jolles è comprendere attraverso quale sostrato e per quali ragioni la forma della fiaba fosse approdata sulle scene teatrali, fino a rivestire un ruolo di primaria importanza all'interno del genere. Per comprendere appieno l'analisi jollesiana è necessaria una breve contestualizzazione storico-critica. Come si sa, il teatro di Carlo Gozzi nacque fondamentalmente come risposta a quello goldoniano. A partire dal 1750 , le commedie di Goldoni iniziavano infatti a riscuotere un notevole successo di pubblico, e l'aristocratico Gozzi mal sopportava che i protagonisti di questo nuovo modo di intendere il teatro fossero vedove scaltre, grezzi gondolieri, giocatori d'azzardo, avvocati e prostitute: dietro alle critiche di matrice stilistica e contenutistica che Gozzi muoveva all'avversario si celava infatti più che altro la preoccupazione per le grandi novità che il teatro goldoniano stava apportando sul piano sociale, 
dal momento che rappresentava vizi privati e pubbliche debolezze della borghesia veneziana, introducendo come protagonisti delle proprie commedie personaggi di ceto basso. Un conservatore come Gozzi non poteva non vedere in tutto ciò una minaccia per l'equilibrio, giudicato già di per sé instabile, della politica veneziana, e un tentativo di sovvertire le istituzioni e le gerarchie sociali. Nelle sue Rime, Gozzi inserì dunque numerosi componimenti satirici e mordaci, volti ad avversare e a demolire le opere di Goldoni (e di Chiari, sullo sfondo), arrivando addirittura a innescare una sorta di tenzone con il poemetto La Tartana degli influssi invisibili per l'anno bisestile 1756 , in cui, facendo una sorta di oroscopo in versi, denigrava lo stile delle commedie goldoniane, lo stucchevole esotismo delle commedie del Chiari, nonché la presenza in quel teatro di protagonisti volgari e borghesi immeritevoli della dignità del palcoscenico. Goldoni accettò la provocazione componendo La tavola rotonda nel I758, un poemetto in cui attaccava a sua volta Gozzi, il quale rincarò la dose iniziale nella satira Il teatro comico all'Osteria del Pellegrino, circolata manoscritta perché censurata, essendo piena di insulti e trivialità. Goldoni accusò nuovamente il colpo, ma decise di rispondere in maniera indiretta, aumentando quella produzione comica che tanto infastidiva il suo detrattore. L'approdo sulla scena di borghesi misantropi come i rusteghi, o dei gondolieri chioggiotti, affermò in maniera perentoria la nuova direzione che la sua riforma, progressista e illuministica, stava imprimendo al teatro. Dopo un nuovo tentativo di demolire il suo avversario con la satira (i dodici canti in ottave de La Marfisa bizzarra, travestimento satirico in chiave boiardesca di personaggi di spicco della Venezia dell'epoca), Gozzi comprese che per contrastare Goldoni avrebbe dovuto impegnarsi sul suo stesso terreno, dando vita a un'alternativa che fosse credibile e accattivante, allo scopo di contrastare efficacemente il realismo goldoniano. L'opportunità più immediata gli era offerta dal suo stesso retroterra culturale: interessato, sin da bambino, alle fiabe e racconti popolari, Gozzi intuì che il realismo goldoniano poteva essere combattuto efficacemente soltanto da un teatro che gli contrapponesse elementi quali il magico, il fiabesco, il meraviglioso, e che al contempo potesse farsi veicolo di quella restaurazione dei costumi di cui le fiabe popolari gli apparivano chiara manifestazione. Per questo motivo, ispirandosi di volta in volta al Pentamerone di Basile, alle Mille e una notte, o al Panchatantra indiano, fino alle fiabe classiche francesi di Perrault, Gozzi iniziò a comporre le sue Fiabe teatrali, con le quali riuscì a mettere in crisi il teatro goldoniano.

Secondo Jolles, l'incontro tra la forma della fiaba e del teatro nella Venezia del secondo Settecento è frutto della situazione individuale e 
storica vissuta dall'autore delle Fiabe teatrali: Gozzi, preoccupato dal potere corrosivo dell'acceso realismo che nutriva il nuovo teatro, trovava nella via obliqua della fiaba esotica e fantastica un modo per arginare le spinte razionalistiche e illuministiche che a suo parere minavano l'ordine costituito. Nella Venezia dell'epoca, il teatro era di gran lunga il mezzo principale per la comunicazione culturale, e il pubblico doveva dunque essere riportato sulla «retta via» proprio grazie al contenuto di queste fiabe, scritte parte in versi e parte in prosa, con alcune scene a soggetto per le maschere, dal tono decisamente anti-illuministico e anti-goldoniano. Attraverso di esse, Gozzi mirava a demolire quelle caratteristiche dell'opera goldoniana che, a parer suo, erano più riprovevoli: da un lato, la mancanza di estetica poetica e la scarsa disciplina stilistica e linguistica ${ }^{\mathrm{I} 2}$, dall'altro la pericolosità sovversiva delle rivendicazioni borghesi e dei programmi di rinnovamento sociale portati avanti dai mercanti, dai professionisti e dai popolani protagonisti delle commedie di Goldoni.

Tuttavia per Jolles la questione fondamentale non porta tanto sulle ragioni interne del teatro di Gozzi, quanto sulla questione dell'intertestualità. Il suo excursus critico individua in primo luogo fonti prettamente letterarie, come i Mille e un giorno di Pétis de La Croix per Turandot, o Basile per Il corvo: si tratta di fiabe desunte dalla letteratura e combinate "con le maschere della commedia dell'arte e tradotte nello stile del suo tempo: un'operazione non molto diversa, in fondo, da quanto Shakespeare aveva fatto con la novella del XVI secolo»" ${ }^{13}$. Ci sono poi fonti in certo senso "miste», vale a dire nutrite da elementi della tradizione orale e da opere a stampa, come è il caso del Re cervo, frutto della contaminazione tra le narrazioni epiche del cantastorie Cigolotti e quelle contenute nel libro riguardante i viaggi e le avventure della principessa di Serendib, che Gozzi poteva aver consultato in una edizione veneziana o francese. Si può individuare infine un terzo tipo di sostrato testuale: sulla scia della tradizionale Commedia dell'Arte, il testo non era scritto per intero ma recitato a partire da un canovaccio e arricchito da elementi fiabeschi in fieri. È il caso ad esempio de L'amore delle tre melarance. Jolles indica per questa commedia una somiglianza d'impianto con la cornice de Lo cunto de li cunti di Basile, ma aggiunge anche che la storia delle tre fanciulle nate dalle melarance potrebbe essere stata desunta da una fiaba popolare diffusamente nota e tramandata oralmente: altrimenti — secondo quanto

I2. Gozzi, membro dell'Accademia dei Granelleschi, era fautore di un linguaggio letterario modellato rigorosamente su quello di Dante, Petrarca e Boccaccio.

I3. A. Jolles, I travestimenti della letteratura, cit., p. I89. 
afferma il critico - non avrebbe potuto attirare il pubblico del teatro di San Samuele ${ }^{\mathrm{I}}$. Il pubblico veneziano mostrò di gradire assai più i drammi fiabeschi di Gozzi rispetto alle commedie borghesi di Goldoni, tanto che i teatri dove le Fiabe teatrali venivano rappresentate erano letteralmente presi d'assalto da un pubblico desideroso di fiabe e di magie, entusiasta di vedere sulle scene fate e giganti, orchi e animali parlanti, maghi e cavalieri di ventura, piuttosto che onesti borghesi e schietti popolani mossi da nobili ideali etici e da concrete richieste di giustizia sociale. Amareggiato, Carlo Goldoni nel 1762 accettò l'invito dei comici italiani in Francia e partì alla volta di Parigi, lasciando così padrone assoluto dei palcoscenici veneziani Carlo Gozzi, il quale continuò a scrivere commedie fiabesche sino al I765. Proprio nella partenza di Goldoni, Jolles individua un momento di svolta nella scrittura delle Fiabe da parte di Gozzi: gli attacchi personali al rivale non erano più necessari, «ma vi era ancora bisogno di combattere ciò che Gozzi considerava il pericolo più grande, in primo luogo le idee degli illuministi francesi [...]. Sulla scena compaiono così caricature impregnate di filosofia francese» ${ }^{15}$. Nella fiaba L'augellin belverde, Gozzi riprende Straparola, Madame d'Aulnoy, suggestioni provenienti da racconti orali, e le piega ai propri scopi comunicativi, facendo «vestire i panni dei personaggi di due fiabe per bambini ai seguaci dei Lumi, che Gozzi vede incarnati in Helvétius, Rousseau e Voltaire», giudicati, nella prefazione, come coloro che «sprezzano e deridono l'umanità col sistema dell'amor proprio, con somma ingratitudine», coloro "che affamati desiderano e lodano i benefizi degli uomini caritatevoli, che fatti ricchi, folleggiano, e vogliono a forza gl'impossibili» ${ }^{16}$.

Si tratta secondo Jolles di una «intuizione audace» ${ }^{17}$, che consente a Gozzi di trasferire la polemica su un piano sociale, permettendo alla morale della fiaba (presente comunque in tutte le pièce dell'autore veneziano) di assumere una dimensione più pregnante e profonda. Sebbene mancanti, per la maggior parte, di vero senso poetico, le fiabe di Gozzi erano tuttavia cariche di colore e di umori: non a caso, come si è detto, furono molto apprezzate dai romantici tedeschi e conobbero una certa fortuna anche in Francia e in Russia, dove registi come Mejerchold e Vachtangov, autore di una memorabile regia di Turandot, apprezzarono Gozzi soprattutto

I4. Il teatro di San Samuele venne costruito a Venezia nel I656, tra il Campo San Samuele e il Campo Santo Stefano, e fu uno dei più prestigiosi e frequentati.

I5. A. Jolles, I travestimenti della letteratura, cit., p. I93.

I6. Opere edite e inedite del Conte Carlo Gozzi. Tomo III, Venezia, dalla Stamperia di Giacomo Zanardi, I8O2, p. 201.

I7. A. Jolles, I travestimenti della letteratura, cit., p. I93. 
per la sua teatralità immaginosa e antinaturalistica. Prendendo le mosse proprio da quest'ultimo dato, ovvero dall'ampio respiro che progressivamente assume il messaggio socio-politico delle fiabe gozziane, e dal loro successo di lunga durata, Jolles conclude il proprio saggio rilanciando la domanda iniziale sulla legittimità della contaminazione tra la fiaba e il teatro. Comprendere se, e in che modo, la lettura di Jolles delle Fiabe di Gozzi e l'intuizione del rapporto dialettico tra il teatro e la fiaba abbiano influenzato la critica successiva è quanto rimane da fare in ulteriori e più approfondite analisi. Si tratta di una sfida non facile, visto il carattere eccentrico (in senso letterale) della riflessione jollesiana nell'orizzonte della critica gozziana, ma proprio per questo forse da tentare, con la consapevolezza di avventurarsi in sentieri dell'italianistica non ancora battuti, che si preannunciano però fecondi di spunti e novità interpretative. 